\title{
Heavy quarkonium production in the Regge limit of QCD: from Tevatron to LHC
}

\author{
Maxim Nefedov*† \\ Samara State University \\ E-mail: nefedovma@gmail.com
}

Vladimir Saleev

Samara State University

E-mail: saleevesamsu.ru

\section{Alexandra Shipilova $§$}

Samara State University

E-mail: alexshipilova@samsu.ru

Heavy quarkonium production in the framework of the non-relativistic quantum chromodynamics and leading order of the parton Reggeization approach at the Tevatron and LHC is discussed. In this note, we compare our predictions for the bottomonium production at the LHC due to the color-singlet approximation of the non-relativistic quantum chromodynamics with CMS and LHCb data. It is found, that in the production of $\Upsilon(1 S)$ states, the color-singlet mechanism is dominating, whereas to describe the data for the inclusive $\Upsilon(2 S)$ and $\Upsilon(3 S)$ production, the coloroctet contributions should be taken into account.

Xth Quark Confinement and the Hadron Spectrum

8-12 October 2012

TUM Campus Garching, Munich, Germany

\footnotetext{
*Speaker.

† Supported by the RFBR, 12-02-31701-mol-a

${ }^{\ddagger}$ Supported by the Russian Ministry of Science and Education, Contract 14.B37.21.1182

${ }^{\S}$ Supported by the RFBR, 12-02-31701-mol-a
} 


\section{Introduction}

The production of heavy quarkonium at hadron colliders provides a useful laboratory for testing the high-energy limit of quantum chromodynamics (QCD) as well as the interplay of perturbative and nonperturbative phenomena in QCD. This high-energy processes are dominated by the multi-Regge final states, when the contribution of partonic subprocesses involving t-channel parton exchanges to the production cross section can become dominant. Then the transverse momenta of the incoming partons and their off-shell properties can no longer be neglected, and we deal with "Reggeized" $t$-channel partons.

We use gauge-invariant approach to introduce off-shell properties of the incoming partons to the matrix element of the hard subprocess, which is based on the gauge-invariant effective action for QCD in the Regge limit [1,2]. This effective field theory incorporates besides ordinary gluons and quarks, also fields of the Reggeized gluons and quarks. Corresponding Feynman rules where obtained in Refs. [2, 3, 4], and they can be used to obtain amplitudes with off-shell incoming partons.

\section{Heavy quarkonium production in the LO PRA.}

In the present note, we study the process of heavy quarkonium production, exploiting the leading order (LO) of parton Reggeization approach (PRA) and non-relativistic QCD (NRQCD) factorization hypothesis $[5,6]$. Working at the $\mathrm{LO}$ in $\alpha_{s}$ and the relative velocity of heavy quarks $v$, we consider the following partonic subprocesses, which describe heavy quarkonium production at high energy:

$$
\begin{aligned}
& R\left(q_{1}\right)+R\left(q_{2}\right) \rightarrow \mathscr{H}\left[{ }^{3} P_{J}^{(1)},{ }^{3} S_{1}^{(8)},{ }^{1} S_{0}^{(8)},{ }^{3} P_{J}^{(8)}\right](p), \\
& R\left(q_{1}\right)+R\left(q_{2}\right) \rightarrow \mathscr{H}\left[{ }^{3} S_{1}^{(1)}\right](p)+g\left(p^{\prime}\right),
\end{aligned}
$$

where $R$ is the Reggeized gluon and $g$ is the Yang-Mills gluon, respectively, with four-momenta indicated in parentheses, $\mathscr{H}[n]$ is the physical charmonium state, produced through the intermediate Fock state of the $q \bar{q}$ pair $\left[{ }^{2 S+1} L_{J}^{(1,8)}\right]$ with the spin $S$, total angular momentum $J$, orbital angular momentum $L$ and in the color-singlet ${ }^{(1)}(\mathrm{CS})$ or in the color-octet ${ }^{(8)}(\mathrm{CO})$ quantum states. The squared amplitudes $\left.\left.\overline{\mid \mathscr{A}(R+R \rightarrow q \bar{q}[2 S+1} L_{J}^{(1,8)}\right]\right)\left.\right|^{2}$ of the relevant subprocesses in the LO PRA have been obtained in the paper [7], and they are shown to be coinciding with our earlier results [8] in the $k_{T}$-factorization approach [9].

In the high-energy factorization, the hadronic cross section is presented as convolution of off-shell partonic cross-section and unintegrated parton distribution functions (uPDF). The best agreement with experiment for the charmonium and bottomonium production, as well as for other processes at low $p_{T}$ [10], was obtained with the use of the KMR [11] procedure, which prescribes how to obtain the uPDFs from the conventional integrated ones. For the more details of the formalism, computational formulas and the fitting procedure for the CO NMEs, see the Ref. [12] and references therein. 

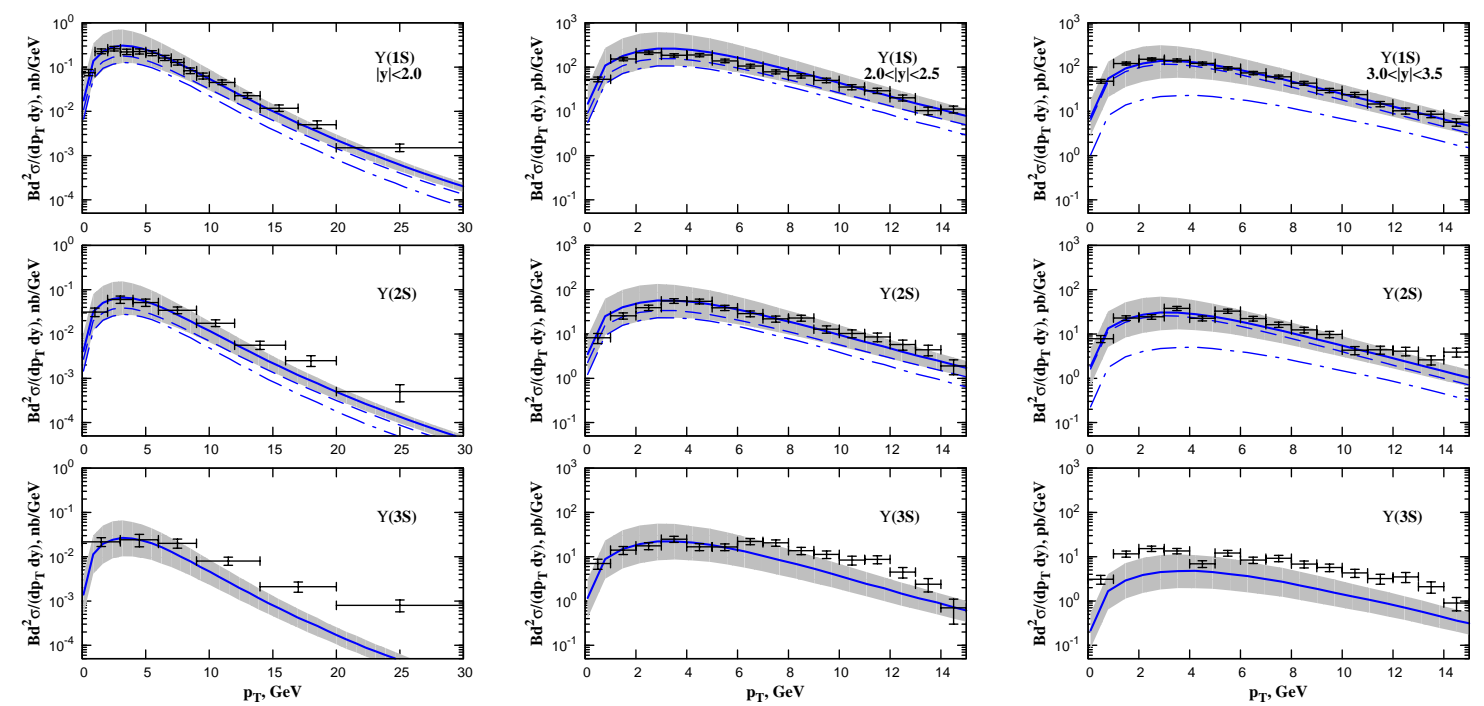

(a)

(b)

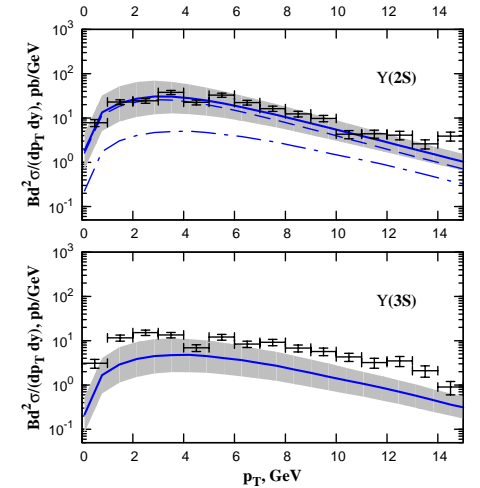

(c)

Figure 1: Comparison of the LO PRA CS model predictions for the inclusive $\Upsilon(n S)$ production with the CMS [16] (a), and LHCb [17] (b, c) data. Dashed lines - direct production, dash-dotted lines - feddown decays contribution, solid lines - their sum. The branching-fractions $B\left(\Upsilon(n S) \rightarrow \mu^{+} \mu^{-}\right)$are included.

\section{Numerical results.}

Our latest numerical results for the prompt charmonium production in the Regge limit of QCD are described in the Ref. [12]. We have shown that it is possible to describe the LHC experimental data on the prompt charmonium production at the $\sqrt{S}=7 \mathrm{TeV}$ in a wide kinematical range $(2<$ $p_{T}<20 \mathrm{GeV}$ and $|y|<3.5$ ) with a good accuracy, using the CO NMEs extracted from Tevatron data at the $\sqrt{S}=1.8 \mathrm{TeV}$ and $1.96 \mathrm{TeV}$. The fitted CO NMEs are also shown to be compatible with NLO collinear parton model results of Ref. [13].

Here we discuss our results for the inclusive $\Upsilon(n S)$ transverse-momentum spectra in the LO PRA and CS approximation of the NRQCD. As in the Ref. [12], here we used the KMR prescription with the MRST-2006 [14] set of the PDFs as the collinear input. The values of the CS NMEs were used the same as in the Ref. [7], taking into account corrected values of branching fractions for the radiative decays of the $\Upsilon(n S)$ and $\chi_{b J}(n P)$ states, accordingly the latest version of Particle Data Group [15]. Due to the lack of space, we do not present in this note our new predictions for the old Tevatron data which show the qualitative features same with the features indicated below for the LHC data.

In the Figure 1, the comparison of the LO PRA prediction on the $\Upsilon(n S)$ production through the CS mechanism with LHC experimental data for inclusive $\Upsilon(n S)$ production in the $p p$-collisions at the $\sqrt{S}=7 \mathrm{TeV}$, measured by the CMS [16] and LHCb [17] collaborations, is presented. The factorization and renormalization scales in this calculation are chosen to be $\mu_{R}=\mu_{F}=\xi M_{T}$ where $M_{T}=\sqrt{M^{2}+p_{T}^{2}}$ is the heavy quarkonium transverse mass, and $\xi$ is varied in the interval $1 / 2<$ $\xi<2$ to estimate the scale-choosing uncertainty of our calculation, and the result of this variation is indicated on the plots by the gray band.

Figure 1 shows that the CS contributions are not sufficient to describe the data for the pro- 
duction of the $\Upsilon(2 S)$ and $\Upsilon(3 S)$ states, whereas the data on the $\Upsilon(1 S)$ production in the region of $p_{T}<15 \mathrm{GeV}$ are described quite well. Moreover, description of $\Upsilon(1 S)$ production in the rapidity interval $3<|y|<3.5$ becomes better, because of the decrease of the fraction of feeddown-decays contribution with rapidity. These features strongly indicate the necessity to include the $\mathrm{CO}$ intermediate state contributions to the calculations. As in the case of the charmonium production $[8,12,13]$, the leading contribution here will be from the $\mathrm{CO}^{3} S_{1}^{(8)}$ intermediate state, which $p_{T^{-}}$ spectrum has the much lower slope, than the slope of the CS contribution. So, as a result of the fit, this contribution should affect the inclusive $\Upsilon(1 S) p_{T}$-spectrum only for the $p_{T}>15 \mathrm{GeV}$, while it's contributions to the production of $\Upsilon(2 S), \Upsilon(3 S)$ and $P$-wave states will be greater. The fit of the CO NMEs for bottomonium production will be the subject of our future study.

\section{References}

[1] L. N. Lipatov, Gauge invariant effective action for high-energy processes in QCD, Nucl. Phys. B 452 (1995) 369 [arXiv: hep-ph/9502308].

[2] L. N. Lipatov, M. I. Vyazovsky, Quasi-multi-Regge processes with a quark exchange in the t-channel, Nucl. Phys. B 597 (2001) 399 [arXiv: hep-ph/ 0009340 ].

[3] V. S. Fadin, L. N. Lipatov, Next-to-leading corrections to the BFKL equation from gluon and quark production, Nucl. Phys. B 477 (1996) 767

[4] E. N. Antonov, L. N. Lipatov, E. A. Kuraev, I. O. Cherednikov, Feynman rules for effective Regge action, Nucl. Phys. B 721 (2005) 111 [arXiv: hep-ph/ 0411185 ].

[5] G. T. Bodwin, E. Braaten, G. P. Lepage, Rigorous QCD analysis of inclusive annihilation and production of heavy quarkonium, Phys. Rev. D 51 (1995) 1125.

[6] F. Maltoni, M. L. Mangano, A. Petrelli, Quarkonium photoproduction at next-to-leading order, Nucl. Phys. B 519 (1998) 361.

[7] B. A. Kniehl, V. A. Saleev, D. V. Vasin, Bottomonium production in the Regge limit of QCD, Phys. Rev. D 74 (2006) 014024 [arXiv: hep-ph/ 0607254 ].

[8] B. A. Kniehl, D. V. Vasin, V. A. Saleev, Charmonium production at high energy in the $k_{T}$-factorization approach, Phys. Rev. D 73 (2006) 074022 [arXiv : hep-ph / 0602179 ].

[9] L.V. Gribov, E.M. Levin, M.G. Ryskin, Semihard processes in QCD, Phys. Rep. 100 (1983) 1.

[10] M. A. Nefedov, N. N. Nikolaev, V. A. Saleev, Drell-Yan lepton pair production at high energies in the Parton Reggeization Approach, (2012) [arXiv : hep-ph/1211.5539].

[11] M. A. Kimber, A. D. Martin, M. G. Ryskin, Unintegrated parton distributions, Phys. Rev. D 63 (2001) 114027 [arXiv:hep-ph/0101348].

[12] V. A. Saleev, M. A. Nefedov, A. V. Shipilova, Prompt J/ $\psi$ production in the Regge limit of QCD: From Tevatron to LHC, Phys. Rev. D 85 (2012) 074013 [arXiv: hep-ph/1201.3464]

[13] M. Butenschoen and B. A. Kniehl, Reconciling J/ $\psi$ production at HERA, RHIC, Tevatron, and LHC with NRQCD factorization at next-to-leading order, Phys. Rev. Lett. 106 (2011) 022003 [arXiv: hep-ph/1009.5662]; World data of J/psi production consolidate NRQCD factorization at NLO, Phys. Rev. D 84 (2011) 051501 [arXiv: hep-ph/1105. 0820].

[14] A. D. Martin, W. J. Stirling, R. S. Thorne, MRST partons generated in a fixed-flavor scheme, Phys. Lett. B 636 (2006) 259 [hep-ph / 0603143 ]. 
[15] K. Nakamura et al. [Particle Data Group], Review of Particle Physics, J. Phys. G 37 (2010) 075021.

[16] V. Khachatryan et al. [CMS Collaboration], Upsilon production cross section in pp collisions at $\sqrt{S}=7 T e V$, Phys. Rev. D 83 (2011) 112004 [arXiv/hep-ex: 1012 . 5545].

[17] R. Aaij et al. [LHCb Collaboration], Measurement of Upsilon production in pp collisions at $\sqrt{S}=7$ TeV, Eur. Phys. J. C 72 (2012) 2025 [arXiv/hep-ex: 1202 . 6579]. 